Revue internationale P.M.E.

Économie et gestion de la petite et moyenne entreprise

\title{
L'utilisation des indices de qualité dans les services : le cas des agences de voyage
}

\section{Nha Nguyen et Michel Marchesnay}

Volume 3, numéro 2, 1990

URI : https://id.erudit.org/iderudit/1007979ar

DOI : https://doi.org/10.7202/1007979ar

Aller au sommaire du numéro

Éditeur(s)

Presses de l’Université du Québec

ISSN

0776-5436 (imprimé)

1918-9699 (numérique)

Découvrir la revue

Citer cet article

Nguyen, N. \& Marchesnay, M. (1990). L'utilisation des indices de qualité dans les services : le cas des agences de voyage. Revue internationale P.M.E., 3(2), 231-248. https://doi.org/10.7202/1007979ar

\section{Résumé de l'article}

Une étude empirique menée auprès de 274 clients de deux agences de voyages a permis de dégager un ensemble d'éléments d'information privilégiés par ces clients dans leur évaluation du service reçu. Ces éléments d'information utilisés comme indices de qualité résident dans la satisfaction du client, sa perception des composantes de la "servuction" telles que l'image de l'entreprise, l'organisation interne, le support physique et le personnel en contact. Les résultats obtenus suggèrent au gestionnaire d'axer sa stratégie d'amélioration de la qualité de son offre de services sur, entre autres, la recherche de la satisfaction du client, l'introduction d'une "culture de qualité" au sein de l'entreprise et le positionnement de l'entreprise par son image de qualité supérieure.
Tous droits réservés @ Presses de l’Université du Québec, 1990
Ce document est protégé par la loi sur le droit d'auteur. L'utilisation des services d’Érudit (y compris la reproduction) est assujettie à sa politique d'utilisation que vous pouvez consulter en ligne.

https://apropos.erudit.org/fr/usagers/politique-dutilisation/ 


\title{
L'utilisation des indices de qualité dans les servi- ces : le cas des agences de voyage
}

\author{
Nha NGUYEN* \\ Université de Moncton, Moncton (N.-B) Canada \\ Michel MARCHESNAY* \\ Université de Montpellier I, Montpellier, France
}

\begin{abstract}
RÉSUMÉ
Une étude empirique menée auprès de 274 clients de deux agences de voyages a permis de dégager un ensemble d'éléments d'information privilégiés par ces clients dans leur évaluation du service reçu. Ces éléments d'information utilisés comme indices de qualité résident dans la satisfaction du client, sa perception des composantes de la «servuction" telles que l'image de l'entreprise, l'organisation interne, le support physique et le personnel en contact. Les résultats obtenus suggèrent au gestionnaire d'axer sa stratégie d'amélioration de la qualité de son offre de services sur, entre autres, la recherche de la satisfaction du client, l'introduction d'une «culture de qualité" au sein de l'entreprise et le positionnement de l'entreprise par son image de qualité supérieure.
\end{abstract}

* Nha Nguyen vient de déposer sa thèse en économie industrielle à l'Université de Montpellier et est professeur à l'Université de Moncton. Michel Marchesnay est docteur d'État en économie industrielle, directeur de l'ERFI et professeur agréé à l'Université de Montpellier I. Il est co-directeur adjoint de la Revue Internationale PME et auteur de plusieurs ouvrages en stratégie et en PME. Adresses: Université de Moncton, Moncton (Nouveau-Brunswick)Canada, E1A 3E9; ERFI, Université de Montpellier I, 14, Cardinalde-Cabrières, 34060 Montpellier, France. Premier manuscrit reçu, février 1990. 


\section{SUMMARY}

This article presents the results of an empirical study on customer perceptions of service quality in two travel agencies, using data collected from 274 clients. The results of the study reveal that service quality is derived principally from customer satisfaction and from factors related to the service delivery process, namely corporate image, internal organization, physical environment and contact personnel. Strategies for implementing and controlling service quality are proposed. Indeed, in controlling for quality it is proposed that managers monitor levels of customer satisfaction and consider quality when developing corporate culture. Furthermore, managers should take service quality intoconsideration when positioning the service firm in its competitive environment.

\section{RESUMEN}

Un estudio empirico en el que han participado 274 clientes de dos agencias de viajes ha permitido destacar una serie de elementos de informacion considerados como primordiales por dichos clientes en su evaluacion del servicio recibido. Estos elementos de evaluacion utilizados como indices de calidad residen en la satisfaccion del cliente, su percepcion de los componentes del sistema de produccion del senvicio tales como la imagen de la empresa, la organizacion interna, el apoyo fisico y el contacto entre el personal. Los resultados del estudio sugieren que la estrategia a utilizar por todo gestionario deseoso de mejorar la calidad de su oferta de senvicios debera centrarse, entre otros, en la busqueda de la satisfaccion del cliente, la introduccion de un «cultivo de la calidad" en el seno de la empresa y el posicionamiento de la empresa por su imagen de calidad superior. 


\section{Introduction}

La qualité est désormais considérée comme une variable qui bâtit la compétitivité de l'entreprise puisqu'elle affecte d'une façon significative le rendement sur le capital investi de celle-ci (Buzzell et Wiersema, 1981). L'entreprise de service n'échappe pas à cette réalité et elle doit intégrer la qualité dans ses systèmes de gestion. Il s'agit là d'une des stratégies essentielles à la réussite, voire même à la survie de l'entreprise de service. Pour celle-ci, l'objectif principal d'une telle stratégie est d'obtenir un avantage distinctif sur ses concurrentes (Marchesnay, 1986).

En raison de la présence souvent nécessaire du client dans la prestation du service, l'entreprise ne peut contrôler la qualité de ce service avant qu'il ne soit «consommé». De plus, le caractère intangible du service rend inopérant le contrôle a posteriori de sa qualité. En fait, il n'est pas toujours possible d'inspecter et mesurer la qualité du service comme un bien à la sortie de son processus de production.

Dans ce contexte, les aspects techniques visant à améliorer la qualité perdent assez souvent leur importance dans le secteur tertiaire. Les entreprises de service soucieuses de la qualité doivent, pour une meilleure gestion de la qualité de leurs offres de services, plutôt privilégier «les approches socio-culturelles en termes de perception de satisfaction de l'utilisateur» (Pavie-Latour,1985). Ainsi, la question de savoir comment le consommateur perçoit la qualité d'un service constitue un intérêt central autant pour les stratèges d'entreprise que pour les théoriciens du comportement du consommateur.

L'objectif du présent article est d'identifier un ensemble de déterminants de la qualité d'une offre de services par le biais d'un modèle explicatif du processus d'évaluation utilisé par le consommateur.

\section{La qualité du service : une notion introuvable?}

\subsection{La prestation du service : une activité particulière}

Les caractéristiques d'un service sont très différentes de celles d'un produit. En fait, on peut associer le service à une démonstration intellectuelle et le produit à une démonstration physique (George et Berry, 1981; Touati, 1984). En plus de son caractère intangible, le service comporte d'autres caractéristiques telles que l'hétérogénéité, la «périssabilité», la simultanéité de sa production et sa consommation, et l'absence de droit de propriété (Judd,1964; Rathmell,1974; Bateson, 1977; Eiglier et Langeard, 1977, 1987; Shostack,1977; Sasser, Olsen et Wyckoff, 1978). Le service est un produit intangible, incorporel, impalpable, qui n'a pas d'existence ou de représentation matérielle, de sorte qu'il peut être consommé, mais ne peut pas être possédé physiquement. Le service est périssable en raison de l'impossibilité de le stocker, car il est perdu s'il n'est pas consommé par le client au moment et à l'endroit 
défini. Le service est hétérogène puisque le résultat de la prestation varie selon le prestataire, selon le client et avec le temps. En général, le service ne peut exister sans la participation du clientà sa production; cela est dû au fait que le service doit souvent être produit et consommé simultanément. De plus, lorsque le client achète un service, il n'acquiert qu'un droit d'accès ou d'usage défini dans le temps et dans l'espace, sans que le droit de propriété soit transféré du prestataire au client. Le service possède donc des attributs souvent difficiles à évaluer par le client même après l'utilisation, de sorte que ses qualités dominantes sont surtout des qualités de croyance (Darby et Kami, 1973) et des qualités d'expérience (Nelson, 1974).

\subsection{II est difficile d'évaluer la qualité}

Dans la littérature du marketing, les études sur la qualité des services sont plutôt rares. La plupart des études recensées offrent des réflexions théoriques n'expliquant que partiellement le concept de qualité perçue et ne présentent pas de vérifications empiriques de leurs propositions conceptuelles. Parallèlement, nous avons identifié trois courants de pensée majeurs dans lesquels s'inscrivent la plupart des travaux de recherche sur l'évaluation de la qualité d'un service par le consommateur.

Selon le premier courant de pensée, le consommateur privilégie l'utilisation des éléments matériels associés au service pour évaluer la qualité de celui-ci. Ces éléments matériels se trouvent soit dans l'environnement physique, soit dans l'équipement ou la machinerie nécessaires à la prestation du service. Il est suggéré de matérialiser ou de tangibiliser le service de manière à le rendre plus concret et attrayant aux yeux du consommateur (Levitt, 1981).

Le deuxième courant de pensée met en relief le rôle déterminant de la performance du personnel en contact dans le processus d'évaluation du consommateur. Les travaux de recherche s'inscrivant dans cette pensée ont proposé des actions articulées autour de la rencontre prestataire-client pour renforcer la qualité du service (Rathmell,1974).

Le troisième courant de pensée, mettant l'accent sur la satisfaction retirée de la prestation par le client, préconise que l'entreprise doit envisager des plans d'action garantissant cette satisfaction pour assurer la qualité de son offre de services, c'està-dire qu'il faut accorder une attention particulière aux facteurs psychologiques, sociologiques et situationnels qui définissent les conditions dans lesquelles se déroule la prestation du service (Lewis et Booms,1983).

Le point de vue exprimé par chaque courant de pensée nous semble restrictif s'il est pris individuellement dans la tentative d'expliquer le processus d'évaluauion du consommateur. Nous pensons qu'il existe une interdépendance entre les éléments d'information proposés par ces courants de pensée. Le consommateur fait appel à un ensemble d'informations découlant des éléments à la fois matériels et immatériels reliés au service et à l'entreprise. Ainsi, chez le client, l'évaluation de la qualité d'une 
offre de services est déterminée par sa perception deséléments tangibles utilisés dans la prestation, la performance du prestataire et le niveau de satisfaction de ses besoins. Cependant, c'est cette demière dimension qui prime aux yeux du client. L'entreprise doit assurer la satisfaction du client à un niveau minimum sur chacun des éléments qui composent l'offre de services et la maintenir dans le temps. Cela s'explique par le fait que le client a tendance à conclure à l'insatisfaction sur l'ensemble de l'offre de services même si une seule composante de celle-ci lui donne une mauvaise impression.

\section{Les modèles déterminants de la qualité des services}

En ce qui concerne la modélisation en matière de qualité des services, l'analyse des trois modèles les plus importants recensés dans la littérature a révélé que les chercheurs ont tenté de décrire simplement les éléments qui composent le concept de qualité perçue.

\subsection{Le modèle de Sasser, Olsen et Wyckoff}

Le modèle développé par Sasser, Olsen et Wyckoff (1978) avance que le consommateur traduit d'abord ses attentes en une série d'attributs désirés du service avant d'évaluer la qualité de celui-ci par le biais d'un processus de comparaison entre ces attributs désirés et les attributs réels constatés lors de la prestation. Selon ces auteurs, les attributs utilisés par le consommateur comme critères d'évaluation sont reliés à la fois au service de base et aux services périphériques. Il s'agit notamment de la sécurité, de la consistance, de la disponibilité et de la variété des services offerts, ainsi que des conditions et du «timing» de la prestation. Malgré l'absence des résultats empiriques, le mérite de ce modèle réside dans sa tentative de décrire la nature des attributs utilisés par le consommateur, de même que la démarche suivie par celui-ci dans son évaluation.

\subsection{Le modèle de Grönroos}

Le modèle proposé par Grönroos (1984) présente trois groupes de facteurs susceptibles d'expliquer la qualité perçue par le client, soit les caractéristiques propres au service, la méthode de prestation et l'image de l'entreprise. Ce modèle souligne par ailleurs le caractère permanent de la dimension institutionnelle de la qualité tout au long du processus d'évaluation, ce qui signifie que le consommateur a toujours une perception quelconque de l'image de l'entreprise même s'il n'a pas d'expérience personnelle avec celle-ci. Le modèle de Grönroos (1984) ne présente qu'une description sommaire des composantes du concept de qualité perçue et de ses facteurs explicatifs. De plus, il n'offre pas suffisamment de précision sur la nature et l'ordre d'importance des relations entre ces composantes. 


\subsection{Le modèle de Parasuraman, Zeithaml et Berry}

Quant au modèle développé par Parasuraman, Zeithaml et Berry (1985), il met en évidence un ensemble de facteurs, appelés déterminants de la qualité perçue du service, tels l'accessibilité, la rapidité, la sécurité, la tangibilité du service, la communication, la compétence, la courtoisie, la crédibilité, la fiabilité du personnel et la compréhension du client. Ces facteurs sont surtout associés au processus de prestation du service.

Nous constatons que ces modèles ont été développés dans une démarche plutôt fragmentaire ne permettant pas de voir la dynamique des interactions entre des composantes de la «servuction», lesquelles interactions jouent un rôle-clé dans la formation de la perception chez le client. Seuls les modèles de Grönroos (1984) et de Parasuraman, Zeithaml et Berry (1985) s'appuient sur quelques vérifications empiriques effectuées auprès des gestionnaires d'entreprise, mais non auprès des clients eux-mêmes. Toutefois, il faut souligner que l'analyse de ces modèles nous a permis de mettre en lumière un ensemble de facteurs susceptibles d'affecter la perception de la qualité du service.

\section{$4 \quad$ Les variables intervenant dans la qualité du service}

Les déterminants de la qualité d'un service résident dans la perception par le client de diverses composantes de la "servuction» et dans la satisfaction qu' il retire de la prestation. Nous proposons à la figure 1, (p. 237), un ensemble de relations causales entre ces éléments et la qualité perçue. Quatre composantes principales de la «servuction» sont considérées dans cette structure de relations : l'image de l'entreprise, l'organisation interne, le support physique et le personnel en contact.

\subsection{L'image de l'entreprise de service est-elle liée à la qualité ?}

L'image d'une entreprise de service perçue par le client est le résultat d'un processus de globalisation. Elle constitue l'identité propre à chaque entreprise, identité perçue par le client par le biais d'un ensemble de caractéristiques associées à la "servuction".

Selon Grönroos (1984), la relation causale entre l'image de l'entreprise et la qualité perçue de son offre de services est une relation réciproque. L'entreprise doit bâtir son image en offrant à sa clientèle des services de qualité. Et inversement, la perception de la qualité des services par le client est largement influencée par l'image de l'entreprise elle-même. A notre avis, la qualité doit être considérée comme variable dépendante et l'image comme variable indépendante dans le contexte d'un 
Figure 1

Modèle explicatif de la qualité perçue d'un service

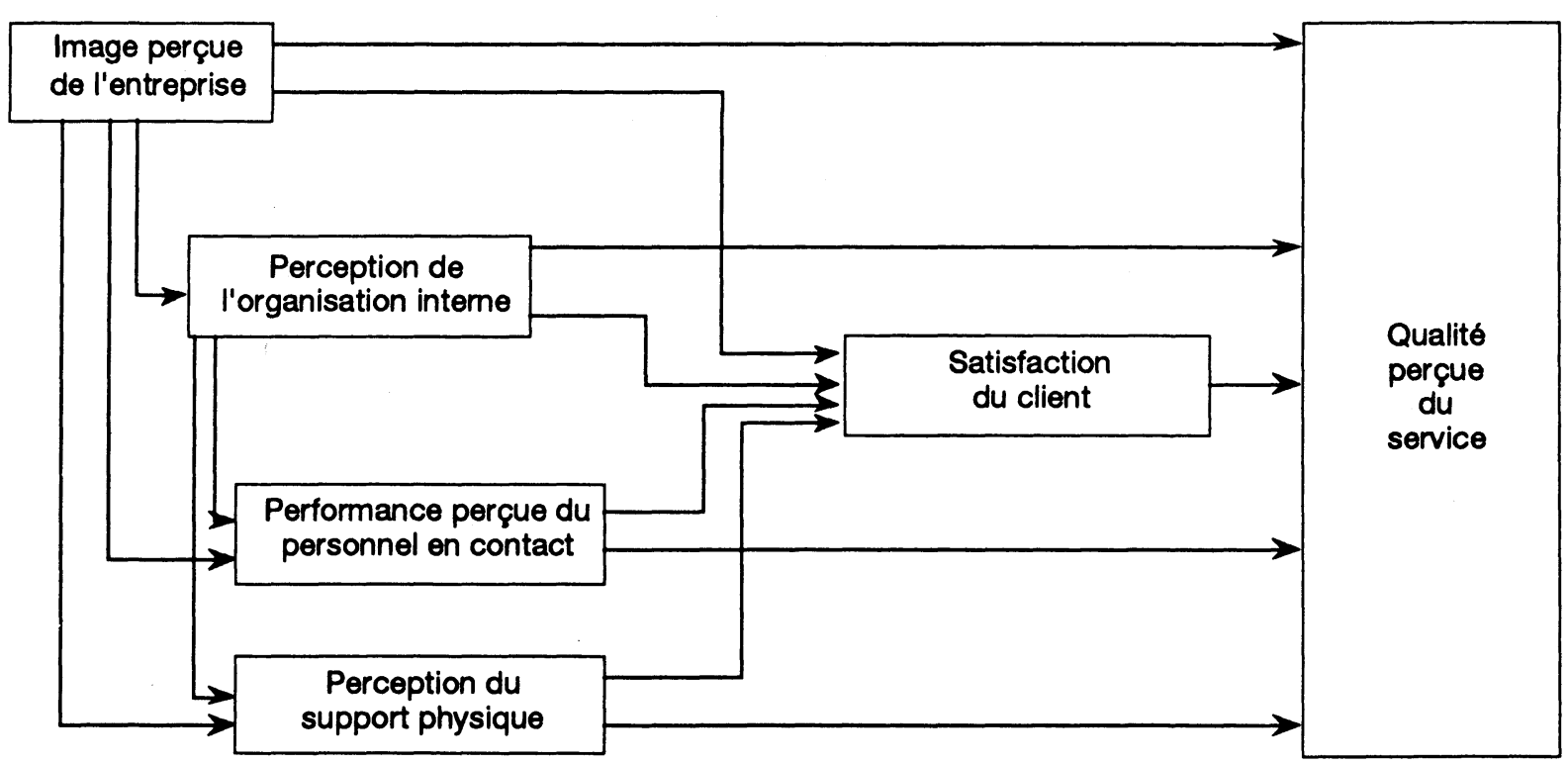


service existant, car la relation causale «qualité-image» définie dans ce sens permet au gestionnaire soucieux de la qualité d'explorer les actions appropriées en sa faveur.

L'image de l'entreprise peut être définie par un ensemble d'attributs tels que le nom de l'entreprise, sa réputation, son caractère distinctif, le prix, la sécurité, l'accessibilité au service, la variêté des services offerts, les bénéfices promis, la garantie du service, la capacité de concurrence, etc.

Hypothèse $\mathrm{H}_{1}$ : Plus la perception par l'utilisateur de services de l'image de l'entreprise est favorable, plus la qualité perçue de l'offre de services est positive.

\subsection{Le système d'organisation Interne de l'entreprise de service Influe-t-ll sur la qualité perçue ?}

Le système d'organisation interne est la partie non-visible du système de «servuction». En règle générale, il s'agit là de l'ensemble des activités non directement reliées à la «servuction». Ce sont notamment des fonctions classiques de l'entreprise et d'autres fonctions spécifiques à la nature du service créé. Elles comprennent, entre autres, les règles de fonctionnement, le personnel de soutien, la procédure de contrôle de la qualité. Le système d'organisation interne est utilisé par le client comme indice de la qualité du service (Eiglier et Langeard 1987; Lovelock, 1984).

Hypothèse $\mathrm{H}_{2}$ : Plus la perception par l' utilisateur de services du système d' organisation interne del' entreprise est favorable, plus la qualité perçue de l'offre de services est positive.

\subsection{Le support physique de la prestation de service, base de sa qualité}

Le service, essentiellement intangible, est souvent évalué par le client par le biais de ses attributs physiques, donc tangibles. Il est souhaitable pour l'entreprise de service de mettre en évidence ses éléments matériels, car le client les utilise comme indices de la qualité (Levitt, 1981; Shostack, 1977). La présence du client dans le processus de «servuction» incite celui-ci à privilégier l'emploi des éléments physiques associés au service pour évaluer sa qualité (Haywood, 1983). Krentler (1981) a, dans une étude empirique, confirmé la relation entre les caractéristiques physiques des services et leur qualité perçue. Il a trouvé que l'intensité de cette relation varie selon le type de bénéfices promis par le service. Le support physique est généralement composé des instruments qui sont tous les objets, meubles ou machines utilisés par le personnel et/ou le client lors de la «servuction», de même que l'environnement, lequel comprend tout ce qui entoure les instruments : le site, les bâtiments, l'aménagement et le décor (Eiglier et Langeard, 1987). 
Hypothèse $\mathrm{H}_{3}$ : Plus la perception parl'utilisateur de services du support physique de l'entreprise est favorable, plus la qualité perçue de l'offre de services est positive.

\subsection{Le personnel en contact, vecteur de la qualité}

La rencontre prestataire-client a un effet important sur l'évaluation du service par le client. En effet, certains auteurs essaient de relier la qualité du service à la structure des interactions entre le client et le «servucteur», en l'occurrence son personnel en contact. Rathmell (1974) associe la qualité à l'acte ou la performance du personnel lors de la prestation du service. Hostage (1975) affirme que le personnel en contact constitue le facteur prépondérant dans la perception de la qualité du service. Par exemple, le client d'un hôtel perçoit la qualité de l'offre par le biais de l'image projetée par son personnel en contact. En conséquence, il est, selon l'auteur, important de contrôler l'attitude et la performance du personnel en contact direct avec le client. Pour Grönroos (1984), c'est la qualité fonctionnelle, dérivée de la rencontre entre le prestataire et le client, qui prime aux yeux de ce dernier.

Solomon, Surprenant, Czepiel et Gutman (1985) proposent aussi que la qualité de la rencontre entre le prestataire et son client détermine dans une large mesure la qualité perçue du service. Le client peut conclure à une mauvaise qualité du service, si le personnel en contact, par son comportement, laisse une mauvaise impression, et cela, en dépit de la qualité supérieure des autres composantes de l'offre de services.

Hypothèse $\mathrm{H}_{4}$ : Plus la perception par l'utilisateur de services de la performance du personnel en contact de l'entreprise est favorable, plus la qualité perçue de l'offre de services est positive.

\subsection{La satisfaction du client, émanation de la qualité perçue}

De nombreux auteurs reconnaissent l'existence d'une relation positive entre la satisfaction et la qualité. En fait, la qualité d'un service est mesurée par la capacité de l'entreprise de combler, voire même de dépasser, les attentes du client (Eiglier et Langeard, 1987; Lewis et Booms,1983; Nightingale,1983; Parasuraman, Zeithaml et Berry, 1985).

Hypothèse $\mathrm{H}_{5}$ : Plus le client est satisfait, mieux il perçoit la qualité de l'offre de services. 


\section{$5 \quad$ Le cas des agences de voyages}

\subsection{Collecte des données}

Dans cette étude, nous avons choisi un secteur des services de conseil destinés aux particuliers, en l'occurrence les agences de voyages, puisque notre intérêt porte uniquement sur l'évaluation faite par le consommateur lui-même. De plus, les services de voyages-vacances exigent généralement un degré de contact élevé entre le prestataire et le client, lequel contact constitue un élément déterminant de la perception de la qualité.

Un questionnaire comprenant 45 variables identifiées suite à l'analyse de la littérature et d'une entrevue de groupe (Cox, Higginbotham et Burton, 1976) fut utilisé comme instrument de mesure de l'évaluation de la qualité, ces variables étant établies sur une échelle de Likert à 7 points. Les questionnaires furent distribués par voie postale à tous les clients actuels (soit 608) de deux agences de voyages ayant les caractéristiques les plus semblables et situées dans une ville d'environ 100,000 habitants; 274 questionnaires complétés furent retournés en l'espace de 2 semaines, ce qui représente un taux de réponse de $45 \%$. En ce qui concerne les 334 nonréponses, les causes sont notamment le refus de réponse (286), l'absence pendant la durée de l'enquête (35) et le questionnaire incomplet (13).

\subsection{Formation des indices}

Cette étape avait pour but de créer les indices représentant les cinq composantes de la «servuction». Chacun des indices est une combinaison à pondération égale des variables qui le composent en raison de l'ignorance de leur degré d'importance les unes par rapport aux autres. L'identification des variables faisant partie d'un concept a été effectuée grâce à l'analyse factorielle des composantes principales tout en respectant la définition du concept lui-même. A la lumière des résultats obtenus par l'analyse factorielle, nous avons choisi les 7 indices les plus représentatifs des cinq composantes de la «servuction» susceptibles d'expliquer la perception de la qualité de l'offre de services. De plus, le calcul des indices s'est fait avec standardisation des nombres de réponses en considérant que les données manquantes sont égales à la moyenne.

\subsection{Fidélité et validité des indices}

Il s'agissait d'un processus de purification des mesures s'appuyant sur deux séries de tests : tests de fidélité et tests de validité. 
- Tests de fidélité : la procédure de vérification de la fidélité des mesures consiste à estimer la variation des scores des différentes variables faisant partie d'un même concept. Dans cette recherche, nous avons eu recours au test d'alpha de Cronbach (Cronbach,1951; Nunnally, 1967 et Peter, 1979) qui est une mesure de la consistance interne. Le tableau 1 montre le coefficient d'alpha de Cronbach calculé pour chaque indice. Puisque la valeur d'alpha de Cronbach est largement supérieure à 0.5 , valeur minimale acceptable pour une recherche exploratoire, tous les indices formés ont été jugés fidèles.

\section{Tableau 1}

Les coefficients d'alpha de Cronbach des indices

\begin{tabular}{|clrrrrr|}
\hline Indices & \multirow{2}{*}{ Noms } & $\begin{array}{c}\text { Nb de } \\
\text { var. }\end{array}$ & $\begin{array}{c}\text { Eten- } \\
\text { due }\end{array}$ & $\begin{array}{c}\text { Moyen- } \\
\text { ne }\end{array}$ & $\begin{array}{c}\text { Ecart- } \\
\text { type }\end{array}$ & $\begin{array}{c}\text { Alpha de } \\
\text { Cronbach }\end{array}$ \\
\hline$I_{1}$ & Image de l'entreprise & 7 & $7-49$ & 31.179 & 11.000 & 0.84 \\
$I_{2}$ & Système d'opération & 10 & $10-70$ & 55.208 & 13.066 & $0.90^{*}$ \\
$I_{3}$ & Procédure de prestation & 3 & $3-21$ & 19.314 & 2.746 & $0.68 *$ \\
$I_{4}$ & Support physique & 7 & $7-49$ & 40.369 & 8.601 & 0.86 \\
$I_{5}$ & Interaction client-personnel & 9 & $9-63$ & 57.951 & 7.984 & $0.92^{* *}$ \\
$I_{6}$ & Interaction client- & & & & & \\
& autres clients & 4 & $4-28$ & 21.979 & 5.294 & $0.76^{* *}$ \\
$I_{7}$ & Satisfaction du client & 3 & $3-21$ & 18.779 & 2.893 & 0.88 \\
\hline
\end{tabular}

- La valeur d'alpha de Cronbach pour $I_{2}$ et $I_{3}$ pris ensemble est égale à $0.83, I_{2}$ et $I_{3}$ représentant conjointement la composante «organisation interne".

** La valeur d'alpha de Cronbach pour $I_{5}$ et $I_{6}$ pris ensemble est égale à $0.81, I_{5}$ et $I_{6}$ représentant conjointement la composante «personnel en contact».

- Tests de validité : nous avons effectué deux tests de validité. Le premier mesure la validité d'inter indice-variable et permet d'indiquer dans quelle mesure les variables désignées contribuent à mesurer le concept représenté par l'indice. Le deuxième évalue la validité convergente, c'est-à-dire le degré de convergence entre deux tentatives de mesure du même concept (Zaltman, Pinson et Angelmar,1973). Dans les deux cas, nous avons utilisé la corrélation de Spearman. Les résultats de ces tests, présentés aux tableaux 2 et 3, (p. 242), ont indiqué que les coefficients de corrélation de Spearman sont tous statistiquement significatifs au seuil de 0.01 , ce qui signifie que les variables désignées contribuent de façon importante à mesurer le concept représenté par l'indice. 
Tableau 2

Les coefficients de corrélation de Spearman inter indice-variable pour la validité des concepts des indices

\begin{tabular}{|c|c|c|c|c|c|c|c|c|c|c|}
\hline Indices & & & & & ariables & & & & & \\
\hline 11 & $\begin{array}{c}x_{1} \\
0.63\end{array}$ & $\begin{array}{c}x_{2} \\
0.67\end{array}$ & $\begin{array}{c}X_{1} \\
0.73\end{array}$ & $\begin{array}{c}X_{4} \\
0.74\end{array}$ & $\begin{array}{c}X_{s} \\
0.67\end{array}$ & $\begin{array}{c}x_{1} \\
0.73\end{array}$ & $\begin{array}{c}X_{7} \\
0.75\end{array}$ & & & \\
\hline $\mathrm{I}_{2}$ & $\begin{array}{c}x_{1} \\
0.67\end{array}$ & $\begin{array}{c}x_{2} \\
0.62\end{array}$ & $\begin{array}{c}X_{j} \\
0.61\end{array}$ & $\begin{array}{c}X_{4} \\
0.68\end{array}$ & $\begin{array}{c}x_{f} \\
0.65\end{array}$ & $\begin{array}{c}X_{0} \\
0.70\end{array}$ & $\begin{array}{c}X_{7} \\
0.66\end{array}$ & $\begin{array}{c}X_{1} \\
0.72\end{array}$ & $\begin{array}{c}x_{0} \\
0.70\end{array}$ & $\begin{array}{c}x_{10} \\
0.68\end{array}$ \\
\hline$I_{3}$ & $\begin{array}{c}x_{1} \\
0.84\end{array}$ & $\begin{array}{c}x_{2} \\
0.60\end{array}$ & $\begin{array}{c}x_{j} \\
0.74\end{array}$ & & & & & & & \\
\hline$I_{4}$ & $\begin{array}{c}x_{1} \\
0.65\end{array}$ & $\begin{array}{c}x_{2} \\
0.79\end{array}$ & $\begin{array}{c}x_{1} \\
0.60\end{array}$ & $\begin{array}{c}X_{4} \\
0.80\end{array}$ & $\frac{x_{5}}{0.77}$ & $\begin{array}{c}x_{0} \\
0.76\end{array}$ & $\begin{array}{c}x_{7} \\
0.63\end{array}$ & & & \\
\hline$I_{5}$ & $\begin{array}{c}X_{1} \\
0.73\end{array}$ & $\begin{array}{c}X_{2} \\
0.61\end{array}$ & $\begin{array}{c}X_{1} \\
0.70\end{array}$ & $\begin{array}{c}X_{4} \\
0.67\end{array}$ & $\begin{array}{c}x_{\delta} \\
0.70\end{array}$ & $\begin{array}{c}X_{8} \\
0.58\end{array}$ & $\begin{array}{c}x_{7} \\
0.60\end{array}$ & $\begin{array}{c}X_{1} \\
0.78\end{array}$ & $\begin{array}{c}X_{1} \\
0.67\end{array}$ & \\
\hline$I_{6}$ & $\begin{array}{c}x_{1} \\
0.68\end{array}$ & $\begin{array}{c}x_{2} \\
0.62\end{array}$ & $\begin{array}{c}x_{3} \\
0.77\end{array}$ & $\begin{array}{c}X_{4} \\
0.83\end{array}$ & & & & & & \\
\hline I & $\begin{array}{c}x_{1} \\
0.89\end{array}$ & $\begin{array}{c}x_{2} \\
0.88\end{array}$ & $\begin{array}{c}X_{1} \\
0.82\end{array}$ & & & & & & - & \\
\hline
\end{tabular}

Tous les coefficients de corrélation de Spearman sont statistiquement significatifs à $p<0.01$.

Tableau 3

Coefficients pour la validité convergente des indices

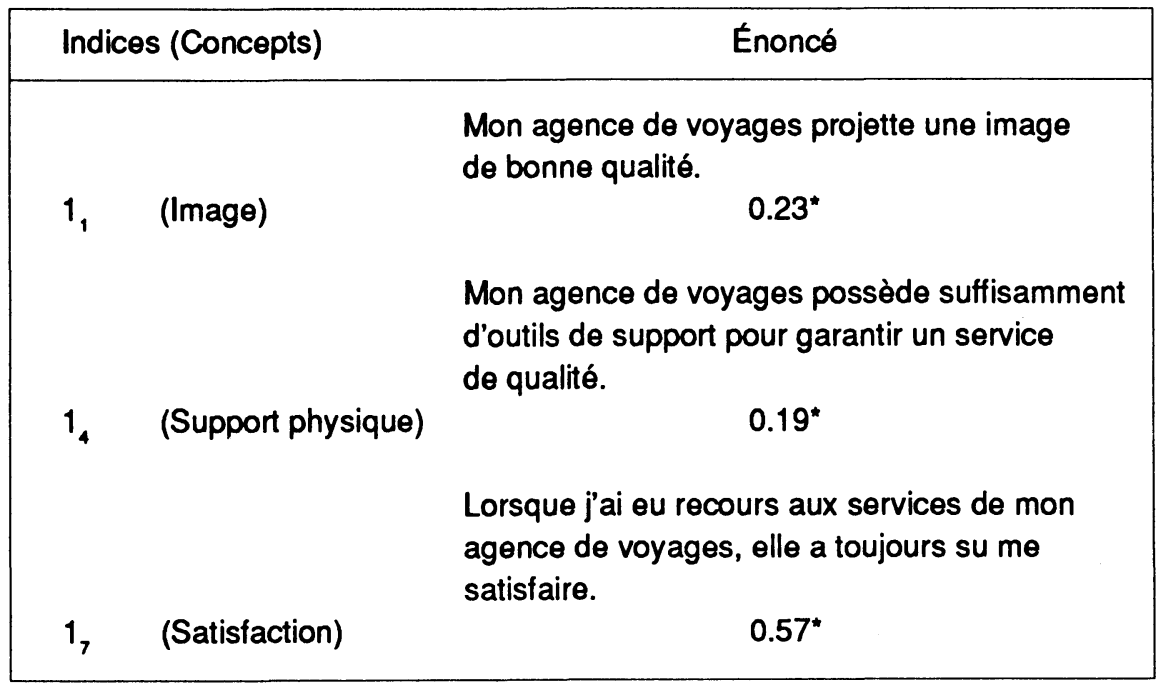

* Significatifs à $p<0.01$ 
A la lumière des résultats des tests de fidélité et de validité, nous avons conclu que les mesures utilisées montrent une consistance interne et que la validité de l'opérationalisation des concepts est vérifiée et concordante avec ce que propose la littérature en ce qui a trait aux déterminants de l'évaluation de la qualité d'un service.

\subsection{Résultats concernant la perception de la qualité du service}

L'ensemble de nos hypothèses forme une structure de causalité entre les 7 indices et la qualité. Pour étudier les relations de cette structure, nous avons fait appel à l'analyse de parcours, technique permettant d'examiner les liens tant directs qu'indirects entre la qualité et les composantes de la «servuction». Nous présentons à la figure $2($ p. 244) la structure vérifiée.

Dans le but de vérifier les hypothèses sur la relation positive entre chacune des composantes de la «servuction» et l'évaluation de la qualité, nous avons calculé l'effet des indices représentant ces composantes sur la qualité en tenant compte des parcours identifiés par la structure de causalité. Les résultats obtenus, résumés au tableau 4, montre que la satisfaction, symbolisée par l'indice 17 , se révèle le facteur le plus important dans l'explication de l'évaluation de la qualité du service par le client. La corrélation entre la satisfaction et la qualité est entièrement contenue dans leur relation directe. Au deuxième rang, on retrouve l'image de l'entreprise (indice $I_{1}$ ). La relation directe entre l'image et la qualité du service étant inexistante, l'influence de l'image sur la qualité perçue se reflète essentiellement, d'une part, dans l'évaluation par le client du système d'organisation interne, du support physique et du personnel en contact, et d'autre part, dans la satisfaction qu'il retire de la consommation du service. L'influence du facteur «personnel en contact» (indices $\mathrm{I}_{5}$ et $\mathrm{I}_{6}$ ) sur la qualité, qui se classe troisième en importance résulte de ses relations à la fois directes et indirectes avec celle-ci. Viennent ensuite les autres composantes l'organisation interne (indices $\mathrm{I}_{2}$ et $\mathrm{I}_{3}$ ) et le support physique (indice $\mathrm{I}_{4}$ ) dont la corrélation avec la qualité est entièrement définie par les facteurs interposés tels que la satisfaction et le personnel en contact. En somme, les résultats ont permis de conclure que les hypothèses ont été confirmées.

Tableau 4

Effet des composantes de la «servuction» sur l'évaluation de la qualité du service

\begin{tabular}{|lcccc|}
\hline $\begin{array}{l}\text { Composantes de la } \\
\text { servuction }\end{array}$ & $\begin{array}{c}\text { Indices } \\
\text { utilisés }\end{array}$ & $\begin{array}{c}\text { Effet } \\
\text { direct }\end{array}$ & $\begin{array}{c}\text { Effet } \\
\text { indirect }\end{array}$ & $\begin{array}{c}\text { Effet } \\
\text { total }\end{array}$ \\
\hline Satisfaction du client & $I_{7}$ & 0.77 & - & 0.77 \\
Image de l'entreprise & $I_{1}$ & - & 0.16 & 0.16 \\
Personnel en contact & 15 & 0.07 & 0.08 & 0.15 \\
& $I_{6}$ & - & 0.08 & 0.08 \\
Organisation interne & $I_{2}$ & - & 0.11 & 0.11 \\
& $I_{3}$ & - & 0.11 & 0.11 \\
Support physique & $I_{4}$ & - & 0.04 & 0.04 \\
\hline
\end{tabular}


Figure 2

Structure de causalité de la qualité du service

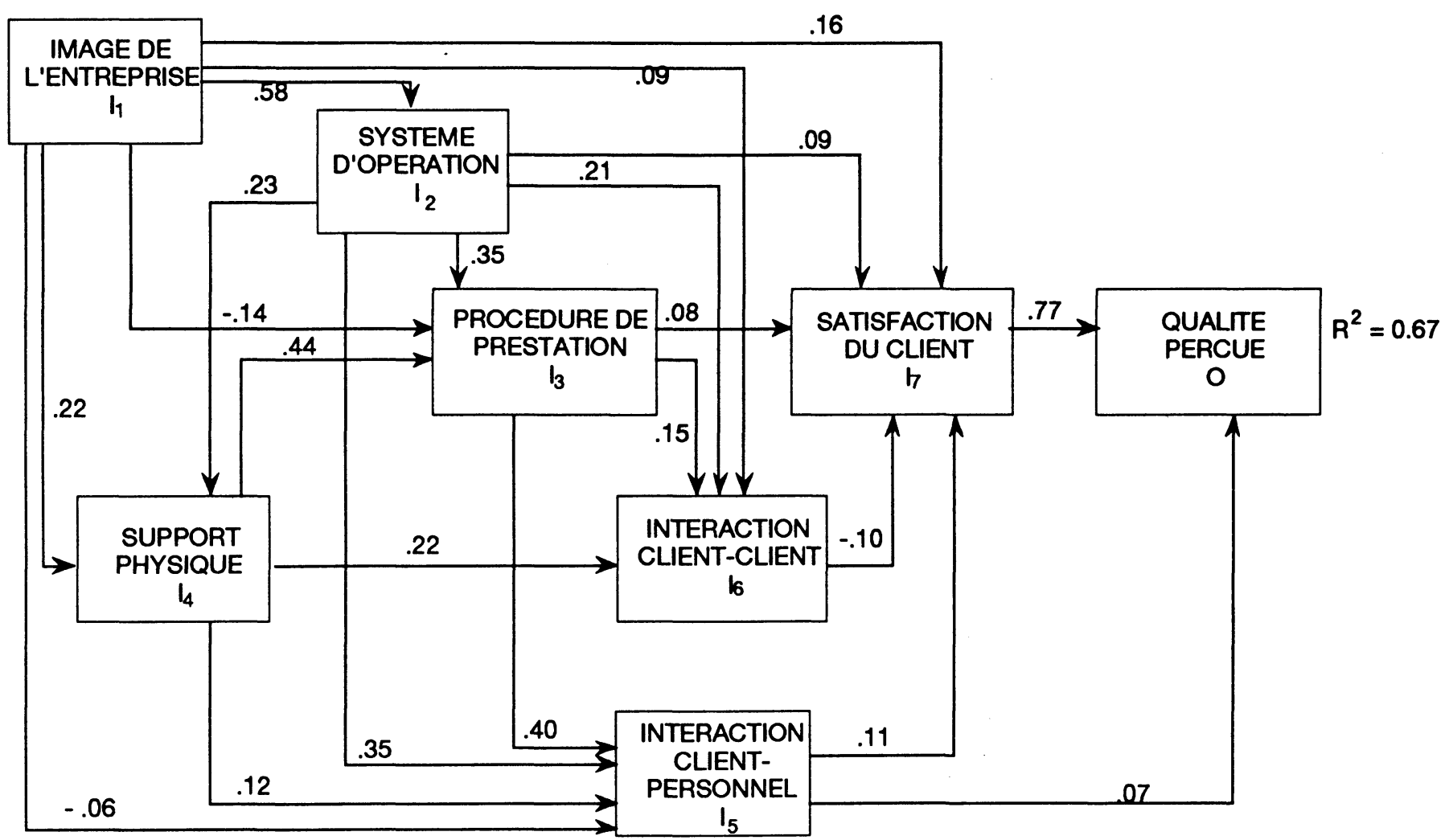

Les coefficients de parcours sont des coefficients de régression standardisés. Ils sont tous statistiquement significatifs à $p<0.05$. 


\section{$6 \quad$ Pour une gestion améliorée de la qualité des services}

Les résultats présentés au tableau 4,(p. 243), révèlent la nette prédominance de la satisfaction du client parmi les facteurs explicatifs de l'évaluation de la qualité du service. En fait, la littérature souligne sans contredit la forte relation positive entre la satisfaction et la qualité (Eiglier et Langeard, 1987; Grönroos, 1984).

\subsection{Le rôle central du client}

Ces résultats suggèrent à l'entreprise de service d'adopter pour son offre de services une conception de la qualité fondée sur la recherche de la satisfaction du client. A cet effet, il est de toute évidence que l'approche «utilisateur», selon laquelle la qualité du service réside dans sa capacité à satisfaire les attentes et besoins du client, doit être privilégiée par le gestionnaire. La gestion de la qualité du service doit à notre avis s'appuyer sur quatre éléments fondamentaux : la stratégie, le système, le personnel et le client lui-même. Le client constitue le point central de cette démarche qui soulève quatre questions fondamentales à toute entreprise de service désirant obtenir un avantage sur ses concurrentes par le biais d'une stratégie axée sur la supériorité de la qualité de son offre de services :

- Quelle est la compréhension, de la part de l'entreprise, des besoins, des attentes et du comportement de la clientèle ?

- L'entreprise a-t-elle élaboré sa stratégie de service en se fondant sur les attentes du client?

- Le système de «servuction» et la procédure de prestation du service sont-ils conçus pour rendre le service plus accessible au client ou pour faciliter la tâche du prestataire?

- Le personnel de l'entreprise, particulièrement celui en contact direct avec le client, est-il bien choisi, formé et motivé pour offrir à ce dernier un service de qualité ?

\subsection{Introduire une «culture de qualité»}

L'entreprise de service doit établir les normes de qualité de son service en s'appuyant sur les attentes et besoins du client, mais non sur les perceptions du gestionnaire. Les normes de qualité aident l'entreprise à clarifier le rôle de chaque participant au processus de production du service et à communiquer ses priorités. L'établissement de ces normes s'inscrit dans une démarche d'institutionnaliser la gestion de la qualité du service pour qu'elle devienne une culture d'entreprise. Cette démarche doit s'implanter à deux niveaux : institutionnel et opérationnel. A cet effet, l'entreprise de service peut faire appel au marketing interne pour stimuler l'adhésion des cadres supérieurs à l'approche de qualité orientée vers le client, pour les convaincre à appuyer les politiques et méthodes de gestion, de planification et de formation. Au niveau opérationnel, le marketing interne permet de faire comprendre 
et accepter par le personnel en contact la procédure à suivre pour respecter les normes de qualité (Grönroos, 1981).

En ce qui concerne le personnel en contact, il doit faire partie intégrante de la stratégie de fidélisation de la clientèle, puisqu'il constitue l'un des facteurs déterminants de l'évaluation de la qualité du service. Il est primordial pour l'entreprise d'élaborer une politique concernant la sélection, la formation, la motivation et la supervision de son personnel. A cet effet, l'entreprise doit définir un style qui lui est propre. Ce style doit guider, entre autres, le choix et la formation de son personnel en contact. L'entreprise doit mettre l'accent sur la précision de la procédure de prestation en définissant clairement le rôle du personnel en contact et celui du client. Elle doit valoriser le travail du personnel en contact qui se trouve dans la plupart des cas aux niveaux hiérarchiques inférieurs malgré sa contribution importante à la réalisation de la mission et des objectifs de l'entreprise. A cet effet, un système de récompense fondé sur le rendement peut être instauré.

\subsection{Soigner l'image de l'entreprise}

Par ailleurs, est-il important de soigner l'image de l'entreprise. Cela implique que celle-ci doit mettre en valeur son personnel en contact et son support physique par le biais d'une politique de communication efficace, car plus le service est intangible, plus le consommateur a tendance à les utiliser comme indices de la qualité. Les actions envisagées en faveur de la qualité du service doivent privilégier l'utilisation des éléments matériels, d'une part, pour informer le client des bénéfices et des exigences du service, et d'autre part, pour créer une atmosphère favorable à la réalisation du service. Par exemple, les agences de voyages peuvent mettre l'accent sur la conception de l'environnement où se déroule la prestation du service pour renforcer leur image institutionnelle.

\subsection{Implications stratégiques}

La présente étude pourra aussi avoir des implications importantes au niveau des décisions stratégiques de l'entreprise, particulièrement en ce qui concerne les stratégies de segmentation de celle-ci et son positionnement sur le marché. L'entreprise de service peut, par exemple, segmenter son marché selon la propension du client à participer à la "servuction" et selon le niveau de qualité du service. Le choix du premier critère a pour effet de faciliter la communication avec la clientèle, tandis que le deuxième suggère à l'entreprise de gérer de façon différente son système de "servuction" selon le niveau de qualité désiré.

En somme, pour mieux atteindre un de ses objectifs fondamentaux, celui de satisfaire le client, la fonction marketing doit mettre l'accent sur :

- la détection des attentes spatio-temporelles de celui-ci;

- la traduction en collaboration avec d'autres fonctions de l'entreprise des attentes du marché en normes de qualité par le biais des caractéristiques perçues par le client. 
L'objectif de cette recherche était d'expliquer l'évaluation de la qualité du service par le client. A cet effet, nous avons pu établir une structure de causalité englobant un ensemble d'éléments dérivés du système de «servuction», à savoir l'image de l'entreprise, l'organisation interne et le support physique de la «servuction», le personnel en contact et la satisfaction du client. Cette structure met en évidence un groupe d'indices de qualité utilisés par le client. Toutefois, il convient de noter que les résultats obtenus dans le cas des agences de voyage ne peuvent en aucun cas être généralisés pour l'ensemble des activité de services. Même pour le secteur de voyages-vacances, les deux agences de voyages choisies ne sont pas nécessairement représentatives, car elles sont différentes des autres tant par leur taille que par la gamme de services offerts. De plus, il est possible que les résultats de l'enquête effectuée durant le congé de Noël ne soient pas représentatifs de toutes les saisons, sachant que les services de voyages-vacances sont généralement soumis à des fluctuations saisonnières.

Par ailleurs, il serait intéressant d'étendre l'étude à d'autres secteurs de service où le niveau de contact entre le client et le prestateur est moins élevé. Ces recherches permettraient de tester la validité externe de la présente recherche.

\section{BIBLIOGRAPHIE}

Bateson, J.E.G., (1977), «Do we need service marketing ?», Marketing Consumer Services New Insights, Marketing Science Institute, Rapport \# 77-115, novembre, p. 1-30.

Buzzell, R.D. et Wiersema, F.D., (1981), «Successful share-building strategies», Harvard Business Review, janvier-février, p. 135.

Cox, K., Higginbotham, J., et Burton, J., (1976), «Applications of focus group interviews in marketing», Journal of Marketing, janvier, p. 77-80.

Cronbach, L.J., (1951), «Coefficient alpha and the internal structure of tests», Psychometrika, septembre, p. 297-334.

Darby, M.R. et Karni, E., (1973), «Free competition and the optimal amount of fraud», Journal of Law and Economics, 16, avril, p. 67-86.

Eiglier, P. et Langeard, E., (1987), Servuction, le marketing des services, Paris, McGraw-Hill.

Eiglier, P. et Langeard, E., (1977), «A new approach to service marketing», Marketing Consumer Services: New Insights, Rapport \#77-115, Cambridge, Mass., Marketing Science Institute.

George, W.R. et Berry, L.L., (1981), «Guidelines for advertising services», Business Horizons, juillet-août, p. 52-56.

Grönroos, C., (1984), «A service quality model and its marketing implications», European Journal of Marketing, vol. 18, no 4, p. 36-44.

Grönroos, C., (1981), «Internal marketing - An integral part of marketing theory», dans Donnelly,J.H.et George,W.R.,(éds.),Marketing of Services, Chicago, American Marketing Association.

Haywood, K.M., (1983), «Assessing the quality of hospitality services», International Journal of Hospitality Management, vol. 2, no 4, p. 165-177.

Hostage, G.M.,(1975), «Quality control in a service business», Harvard Business Review, juilletaoût, p. 98-106. 
Judd, R.C., (1964), «The case for redefining services», Journal of Marketing, janvier, 28, p. 58-59.

Krentler, K.A., (1981), «Empirical investigation of the use of tangibles cues in the marketing of consumer services», Thèse doctorale, University of Kentucky.

Levitt, T.,(1981), «Marketing intangible products and product intangibles», Harvard Business Review, mai-juin, p. 94-102.

Lewis, R. et Booms, B., (1983), «The marketing aspects of service quality», Proceedings AmericanMarketing Association: Emerging Perspectives on Services Marketing. L. Berry, G.L. Shostack et G. Upah (éds.), p. 99-104.

Lovelock, C.H. (1984), Services Marketing, N.J., Englewoods, Prentice-Hall.

Marchesnay, M., (1986), La stratégie, du diagnostic à la décision industrielle, Paris, Chotard et Associés Editeurs.

Nelson, P.(1974), «Advertising and information», Journal of Political Economy, vol. 82, juilletaoût, p. 729-754.

Nightingale, M., (1983), «Defining quality for a quality assurance program... A study of perceptions», PQCS Management Consultants, Londres, Angleterre.

Nunnally, J. (1967), Psychometric Theory, New York, McGraw-Hill.

Parasuraman A., Zeithaml, V.A. et Berry, L.L., (1985), «A conceptual model of service quality and its implications for future research", Journal of Marketing, automne, vol. 49, no 4, p. 41-50.

Pavie-Latour, B., (1985), «La compétitivité par la qualité», Revue française de gestion, no 52, juin-juillet, p. 84-92.

Peter, P., (1979), «Reliability: A review of psychometric basics and recent marketing practices», Journal of Marketing Research, février, p. 6-17.

Rathmell, J., (1974), Marketing in the Service Sector, Cambridge, Mass., Winthrop Publishers Inc., .

Sasser, W.E., Olsen, P. et Wyckoff, D., (1978), Management of Service Operations, Texts, Cases and Readings, Boston, Mass., Allyn et Bacon.

Shostack, G. L., (1977), «Breaking free from product marketing», Journal of Marketing, vol. 41, avril, p. 77-80.

Solomon, M. R, Surprenant, C., Czepiel, J.A. et Gutman, E.G., (1985), «A role theory perspective on dyadic interactions: The service encounter», Journal of Marketing, vol.49, hiver, p. 99-111.

Touati, O., (1984), «Concept de service et marketing de l'incorporel», Thèse doctorale, Université Paris IX Dauphine, Paris.

Zaltman, G., Pinson, C. et Angelmar, R.,(1973), Metatheory and Consumer Research, Dryden Press, Hindsdale, Ill. 


\section{CORRECTION}

Le lecteur est prié de noter que les résultats présentés aux tableaux $1,2,3$ et 4 et à la figure 2 de l'article intitulé «L'utilisation des indices de qualité dans les services : le cas des agences de voyages» par Nha Nguyen et Michel Marchesnay, paru en 1990 dans la Revue Internationale P.M.E., vol. 3, $\mathrm{n}^{\circ} 2$, p. 232-248, comportent des erreurs. En fait, ces résultats proviennent d'une autre base de données traitant des institutions financières, au lieu de celle des agences de voyages. La confusion s'est produite lors de la manipulation des fichiers de données. Les auteurs en assument l'entière responsabilité et présentent leurs excuses aux lecteurs de la Revue.

Nous présentons ici les nouveaux tableaux $1,2,3$ et 4 et la nouvelle figure 2 , véritables résultats du secteur des agences de voyages.

Le lecteur intéressé aux résultats du secteur des services financiers peut consulter l'article présenté par le premier auteur, intitulé «Un modèle explicatif de l'évaluation de la qualité d'un service: une étude empirique», et paru en 1991 dans la revue Recherche et Application en Marketing, vol. VI, $\mathrm{n}^{\circ}$ 2, p. 83-98.

TABLEAU 1

Les cefficients d'alpha de Cronbach des indices

\begin{tabular}{clrrrrr}
\hline Indices & Noms & Nb de var. Étendue & Moyenne Écart-type $\begin{array}{c}\text { Alpha de } \\
\text { Cronbach }\end{array}$ \\
\hline $\mathrm{I}_{1}$ & Image de l'entreprise & 10 & $16-70$ & 57,019 & 8,310 & 0,76 \\
$\mathrm{I}_{2}$ & Système d'opération & 5 & $5-35$ & 29,679 & 4,873 & $0,83^{*}$ \\
$\mathrm{I}_{3}$ & Procédure de prestation & 6 & $15-42$ & 36,250 & 4,894 & $0,73^{*}$ \\
$\mathrm{I}_{4}$ & Support physique & 8 & $8-56$ & 38,408 & 9,868 & 0,89 \\
$\mathrm{I}_{5}$ & Interaction & & & & & \\
& client-personnel & 9 & $29-63$ & 56,889 & 5,392 & $0,81^{* *}$ \\
$\mathrm{I}_{6}$ & Interaction & & & & & \\
& client-autres clients & 5 & $8-35$ & 25,942 & 5,594 & $0,70^{* *}$ \\
$\mathrm{I}_{7}$ & Satisfaction du client & 3 & $3-21$ & 18,429 & 3,065 & 0,91 \\
\hline
\end{tabular}

* La valeur d'alpha de Cronbach pour $I_{2}$ et $I_{3}$ pris ensemble est égale à $0,83, I_{2}$ et $I_{3}$, représentant conjointement la composante « organisation interne ».

** La valeur d'alpha de Cronbach pour $\mathrm{I}_{5}$ et $\mathrm{I}_{6}$ pris ensemble est égale à $0,81, \mathrm{I}_{5}$ et $\mathrm{I}_{6}$, représentant conjointement la composante " personnel en contact». 
TABLEAU 2

Les cœfficients de corrélation de Spearman inter indice-variable pour la validité des concepts des indices

\begin{tabular}{lcccccccccc}
\hline Indices & \multicolumn{10}{c}{ Variables } \\
\hline $\mathrm{I}_{1}$ & $\mathrm{X}_{1}$ & $\mathrm{X}_{2}$ & $\mathrm{X}_{3}$ & $\mathrm{X}_{4}$ & $\mathrm{X}_{5}$ & $\mathrm{X}_{6}$ & $\mathrm{X}_{7}$ & $\mathrm{X}_{8}$ & $\mathrm{X}_{9}$ & $\mathrm{X}_{10}$ \\
& 0,62 & 0,47 & 0,65 & 0,67 & 0,42 & 0,56 & 0,52 & 0,59 & 0,59 & 0,55 \\
$\mathrm{I}_{2}$ & $\mathrm{X}_{1}$ & $\mathrm{X}_{2}$ & $\mathrm{X}_{3}$ & $\mathrm{X}_{4}$ & $\mathrm{X}_{5}$ & & & & & \\
& 0,71 & 0,79 & 0,81 & 0,75 & 0,72 & & & & & \\
$\mathrm{I}_{3}$ & $\mathrm{X}_{1}$ & $\mathrm{X}_{2}$ & $\mathrm{X}_{3}$ & $\mathrm{X}_{4}$ & $\mathrm{X}_{5}$ & $\mathrm{X}_{6}$ & & & & \\
& 0,64 & 0,77 & 0,70 & 0,69 & 0,60 & 0,61 & & & & \\
$\mathrm{I}_{4}$ & $\mathrm{X}_{1}$ & $\mathrm{X}_{2}$ & $\mathrm{X}_{3}$ & $\mathrm{X}_{4}$ & $\mathrm{X}_{5}$ & $\mathrm{X}_{6}$ & $\mathrm{X}_{7}$ & $\mathrm{X}_{8}$ & & \\
& 0,67 & 0,82 & 0,71 & 0,80 & 0,76 & 0,72 & 0,63 & 0,59 & & \\
$\mathrm{I}_{5}$ & $\mathrm{X}_{1}$ & $\mathrm{X}_{2}$ & $\mathrm{X}_{3}$ & $\mathrm{X}_{4}$ & $\mathrm{X}_{5}$ & $\mathrm{X}_{6}$ & $\mathrm{X}_{7}$ & $\mathrm{X}_{8}$ & $\mathrm{X}_{9}$ & \\
& 0,67 & 0,67 & 0,67 & 0,70 & 0,66 & 0,74 & 0,71 & 0,53 & 0,62 & \\
$\mathrm{I}_{6}$ & $\mathrm{X}_{1}$ & $\mathrm{X}_{2}$ & $\mathrm{X}_{3}$ & $\mathrm{X}_{4}$ & $\mathrm{X}_{5}$ & & & & & \\
& 0,76 & 0,59 & 0,51 & 0,74 & 0,61 & & & & & \\
$\mathrm{I}_{7}$ & $\mathrm{X}_{1}$ & $\mathrm{X}_{2}$ & $\mathrm{X}_{3}$ & & & & & & & \\
& 0,92 & 0,90 & 0,89 & & & & & & & \\
\hline
\end{tabular}

Tous les cœfficients de corrélation de Spearman sont statistiquement significatifs à $\mathrm{p}<0,01$.

\section{TABLEAU 3}

\section{Cofficients pour la validité convergente des indices}

\begin{tabular}{ll}
\hline Indices (Concepts) & Énoncé \\
\hline $\mathrm{I}_{1}$ (Image) & $\begin{array}{l}\text { Mon agence de voyages projette une image de bonne qualité. } \\
0,39^{*}\end{array}$ \\
& $\begin{array}{l}\text { Mon agence de voyages possède suffisamment d'outils } \\
\text { Ie support pour garantir un service de qualité. } \\
\text { I (Support physique) }\end{array}$ \\
& $\begin{array}{l}\text { Lorsque j'ai eu recours aux services de mon agence } \\
\text { Ie voyages, elle a toujours su me satisfaire. }\end{array}$ \\
\hline
\end{tabular}

* Significatifs à $\mathrm{p}<0,01$ 
TABLEAU 4

Effet des composantes de la servuction sur l'évaluation de la qualité du service

\begin{tabular}{lcccc}
\hline $\begin{array}{l}\text { Composantes de la } \\
\text { servuction }\end{array}$ & $\begin{array}{l}\text { Indices } \\
\text { utilisés }\end{array}$ & $\begin{array}{c}\text { Effet } \\
\text { direct }\end{array}$ & $\begin{array}{c}\text { Effet } \\
\text { indirect }\end{array}$ & $\begin{array}{c}\text { Effet } \\
\text { total }\end{array}$ \\
\hline Satisfaction du client & $\mathrm{I}_{7}$ & 0,87 & - & 0,87 \\
Image de l'entreprise & $\mathrm{I}_{1}$ & - & 0,52 & 0,52 \\
Personnel en contact & $\mathrm{I}_{5}$ & 0,10 & 0,07 & 0,17 \\
Organisation interne & $\mathrm{I}_{6}$ & 0,09 & 0,05 & 0,14 \\
& $\mathrm{I}_{2}$ & - & $-0,05$ & $-0,05$ \\
Support physique & $\mathrm{I}_{3}$ & - & 0,08 & 0,08 \\
\hline
\end{tabular}


FIGURE 2

Structure de causalité de la qualité du service

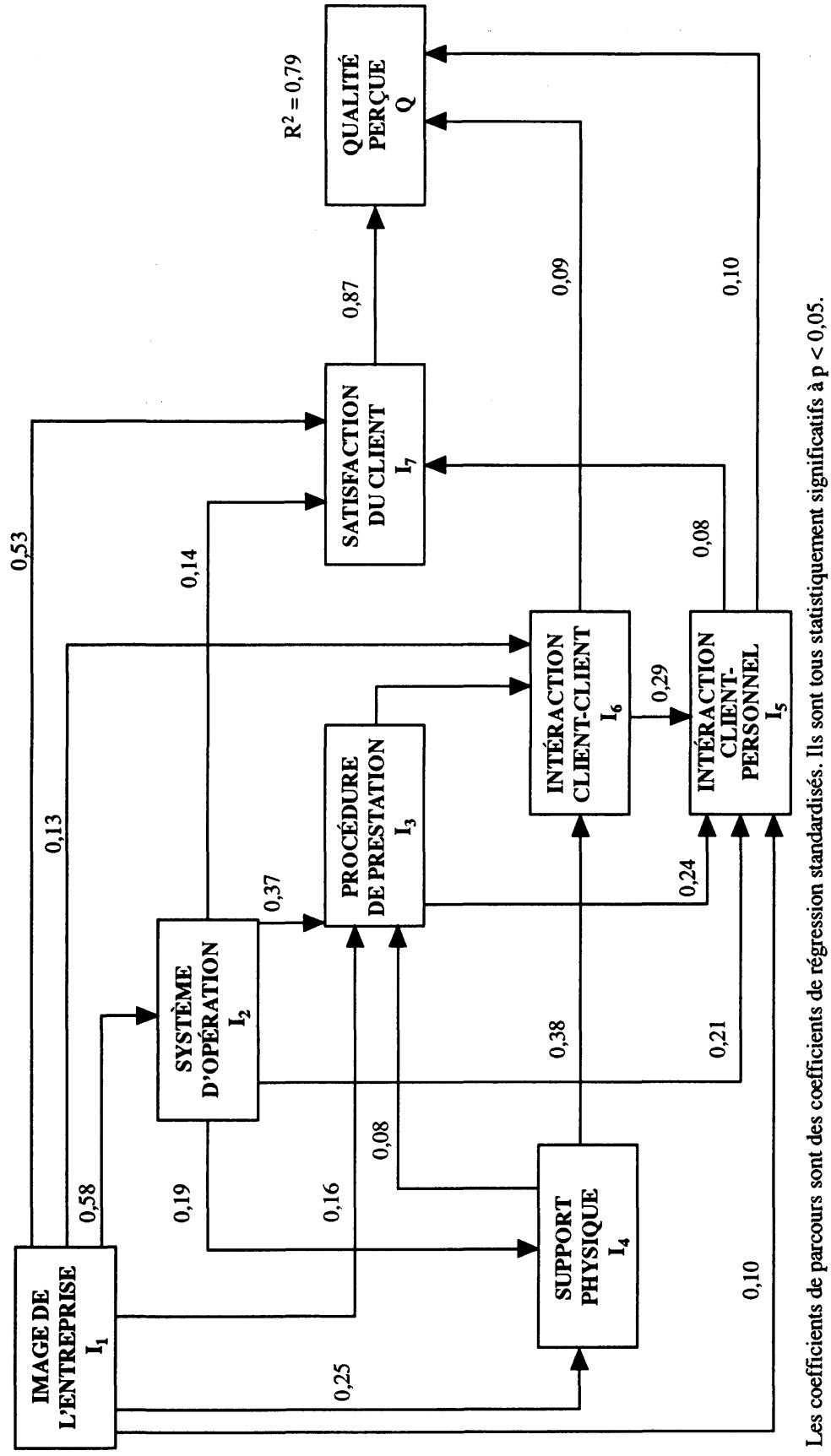

Revue internationale P.M.E., vol. 4, $\mathrm{n}^{\circ}$ 2, 1991 